\title{
MONITORAMENTO COGNITIVO NA PRODUCAO DA FALA E DA ESCRITA
}

\author{
Eneida Werner
}

Eneida Werner é doutoranda em Estudos da Linguagem da PUC-Rio.

E-mail: eneida_werner@hotmail.com

\begin{abstract}
Resumo
O presente artigo tem como objetivo pontuar o conceito de auto monitoramento cognitivo, com base na literatura especifica da area. Relatamos os mecanismos e sistemas de detecção e correção de erros e a Teoria do Loop Perceptual de Levelt (1983, 1989) na produção da fala e tentamos estabelecer um paralelo com produção escrita.
\end{abstract}

\begin{abstract}
The present article aims at pointing out the concept of cognitive self monitoring based on the specific literature in the area. We relate the mechanisms and systems for error detection and correction and the Theory of the Perceptual Loop from Levelt $(1983,1989)$ in speech production and we try to establish a parallel to writing production.
\end{abstract}

\section{1) Apresentação}

Intuitivamente sabemos que temos a capacidade de detectar os nossos erros de fala e escrita e somos capazes de acionar mecanismos que neutralizem seus efeitos. Esse processo de mapeamento, percepção de falha e autocorreção é o que entendemos como o monitoramento cognitivo na produção de fala e escrita.

É um dos estágios da produção linguística descritos por Levelt (1989) que são, bem sinteticamente, quatro: a conceptualização e a formulação da mensagem, a articulação fonológica e o automonitoramento do sujeito produtor.

O presente trabalho tem como objetivo focalizar apenas no recorte referente ao monitoramento. Pretende-se relacionar esse processo cognitivo com a Teoria do Loop Perceptual (Levelt, 1983, 1989) com vistas a aprofundar o entendimento do papel desempenhado pelo monitoramento na produção de linguagem oral e escrita.

Entretanto, em primeiro lugar, é necessário caracterizar o processo de detecção de erros e reparações , a Teoria do Loop Perceptual e o processo de revisão e feedback.

\section{2) Detecção e reparação de erros}

Na produção da fala, o processo de automonitoramento desempenha um papel crucial. As finalidades do automonitoramento são de observar, detectar e corrigir erros da produção da fala ou partes dela ou da saída do enunciado que não estejam em acordo com a intenção de comunicação do falante ou com sua compreensão linguística ou seu padrão linguístico (Postma \& Kolk, 1993 apud Postma, 2000). 
Nesse sentido, o monitor funciona como um mecanismo que ouve o feedback interno ou externo do enunciado produzido, o compara com os dados de saída da produção de fala, identifica inconsistências e, em seguida, procede a computação de correções usando uma espécie de cópia duplicada da informação linguística inicialmente disponível e a envia para o sistema motor executivo, que avalia a relevância da informação a ser corrigida. Avaliado esse critério e reavaliados os dados de entrada para a computação linguística, retoma-se assim sucessivamente, uma nova etapa no processo de reconhecimento de erros (error detection, Levelt, 1983, 1989), sejam estes de nível motor, linguístico ou cognitivo. Levelt (1983) considera que o processo de detecção de erros e reparações estão sujeitos a segmentações do enunciado original, a partir do momento da detecção do erro, seguido de uma fase de edição de um novo enunciado com uso de um termo de edição e a correção ou reparação propriamente dita, quando então é retomado o fluxo do discurso.

Para ilustrar esse processo, reproduzimos, na Figura 1, a estrutura de reparação de erros de fala, segundo Levelt (1983).

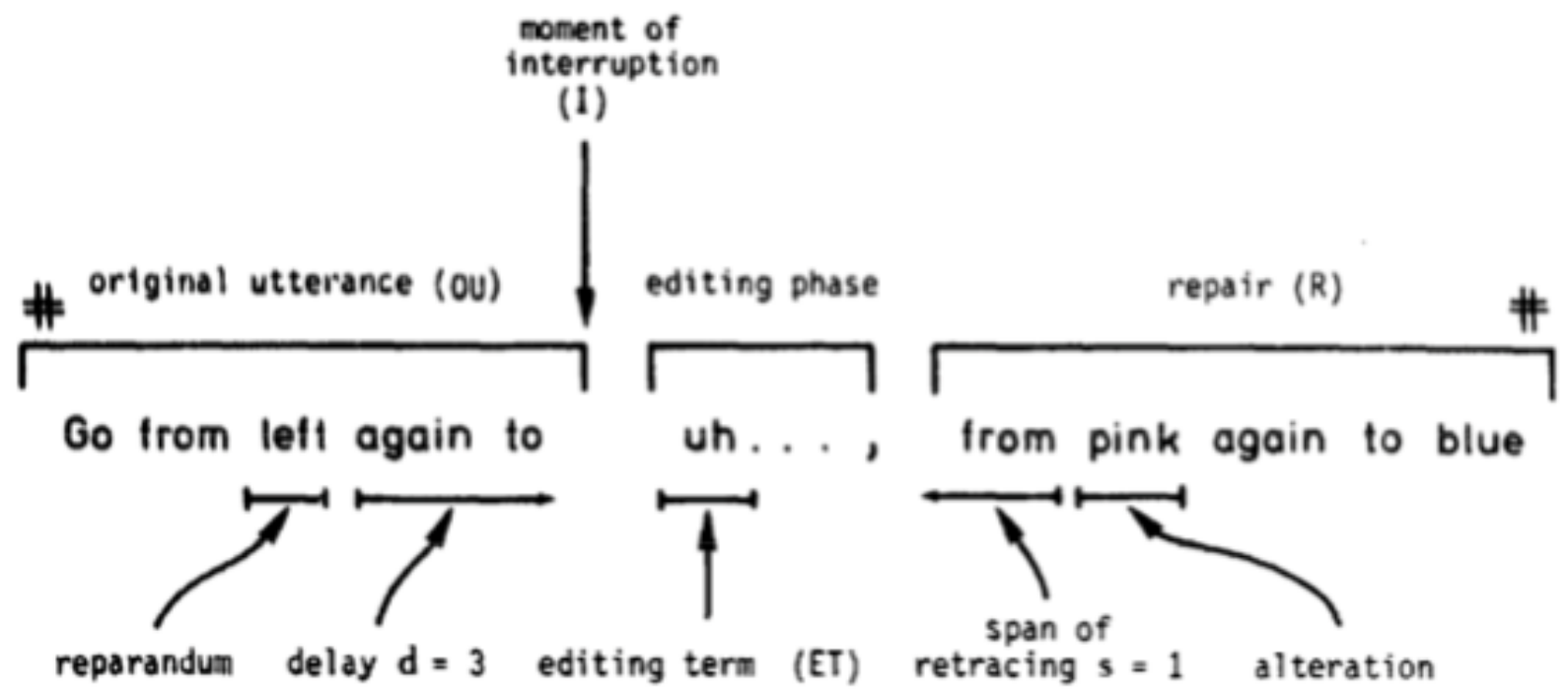

Figura 1: A estrutura da reparação de erros de fala (Levelt, 1983)

Os sistemas de detecção de erros são caracterizados por três níveis hierarquicamente organizados: o nível motor, de caráter mais superficial relacionado à detecção de erros de digitação; o nível linguístico, no qual são detectados erros 
objetivos que comprometem a estrutura sintática do enunciado produzido e o nível cognitivo, relacionado à detecção de erros de caráter subjetivo referentes à estrutura do pensamento e que se relacionam à capacidade de tradução de uma representação mental para um código linguístico. Dos três níveis de sistemas de detecção de erros conhecidos, o sistema motor é um nível hierárquico mais baixo do que os níveis linguístico e cognitivo. Quando o sistema de detecção de erros é ativado, ativa-se o processo de automonitoramento.

Esses três níveis de detecção de erros compartilham os mesmos recursos de memória e competem entre si na alocação desses recursos. É amplamente aceito por pesquisadores da área de cognição que a memória de trabalho tem capacidade limitada (Baddeley et al., 1998, Baddeley \& Hitch, 1974, Ericsson \& Kintsch, 1995). Portanto, o funcionamento eficiente requer necessariamente bom aproveitamento e distribuição equilibrada dos recursos de memória envolvidos na produção linguística. Segundo Postma (2000), é plausível supor que o monitoramento é um processo de recursos limitados de memória. Uma vez que, sendo alocados recursos para desempenho de outras atividades, o processo de monitoramento não conseguirá operar satisfatoriamente nos três níveis que o constitui.

Para Levelt (1983) os indivíduos podem atuar sobre o sistema de monitoramento, sugerindo, com isso, que o monitoramento é um processo mental consciente. Levelt aprofundou a pesquisa do monitoramento em estudos que buscavam capturar dados do comportamento de produção com base no critério de segmentações (interruptions) utilizados pelos sujeitos produtores. O quadro abaixo, quantifica as segmentações realizadas por participantes de seu experimento de monitoramento. Verifica-se o tipo de interrupção (a) apropriação e (b) correção de erros (appropriateness and error repair) nos níveis imediato e posterior (immediate/delayed) e nos subníveis, a localização onde esses erros foram detectados (within word/after word). Note-se o aumento significativo de correções dos níveis imediato e posterior no subnível após a palavra. Depreende-se da tabela abaixo, que a busca por erros correlaciona-se positivamente com o número de segmentações. A interrupção é relacionada ao momento de atenção e percepção de inadequação entre o texto produzido e a representação mental do autor. 


\section{Within-word and after-wnrd interruptions for appropriateness and error repairs}

\begin{tabular}{|c|c|c|c|c|c|c|}
\hline \multirow{3}{*}{$\begin{array}{l}\text { Type of } \\
\text { repair }\end{array}$} & \multicolumn{4}{|c|}{ Interruption } & \multirow{3}{*}{$\begin{array}{l}\text { Average delay } \\
\therefore \text { in syllables }\end{array}$} & \multirow[t]{3}{*}{ Toral } \\
\hline & \multicolumn{2}{|c|}{ Immediate } & \multicolumn{2}{|c|}{ Delayed } & & \\
\hline & $\begin{array}{l}\text { Within- } \\
\text { word }\end{array}$ & $\begin{array}{l}\text { After- } \\
\text { word }\end{array}$ & $\begin{array}{l}\text { Within- } \\
\text { sord }\end{array}$ & $\begin{array}{l}\text { After- } \\
\text { word }\end{array}$ & & \\
\hline A-repair & $20(7 \%)$ & $155(53 \%)$ & $10(3 \%)$ & $105(36 \%)$ & 2.85 & $290(100 \%)$ \\
\hline E-repair & $91(23 \%)$ & $193(48 \%)$ & $i 9(5 \%)$ & $96(24 \%)$ & 3.79 & $399(100 \%)$ \\
\hline
\end{tabular}

Figura 2: Interrupções dentro e após a palavra, Levelt (1983)

3) Teoria do Loop Perceptual (Levelt, 1983, 1989)

A teoria de automonitoramento verbal mais influente e detalhada é a teoria proposta por Levelt $(1983,1989)$ e está relacionada à fala. A Teoria do Loop Perceptual baseia-se no sistema de compreensão linguístico como fonte de realimentação do sistema. Sugere um monitor central, localizado no conceptualizador da mensagem e admite a existência de um monitoramento externo e interno. O monitoramento externo, de acordo com esse modelo, é o monitoramento do enunciado depois da articulação fonológica e prossegue de forma cíclica e contínua através do loop auditivo. Um sinal acústico entra no sistema auditivo e é, então, processado pelo sistema de compreensão da fala, onde a informação é analisada e, em seguida, reenviado para o conceptualizador. O monitoramento interno exerce controle sobre a produção de fala e acontece antes que a fase de articulação do enunciado tenha acessado níveis mais abstratos, como a informação fonológica. A função do loop interno é mapear o plano do discurso antes de chegar à articulação. Finalmente, há um loop conceitual entre a mensagem pré-verbal e o conceptualizador que checa a adequação semântica do enunciado para lançar a mensagem para os sistemas de desempenho e finalmente para a articulação. 


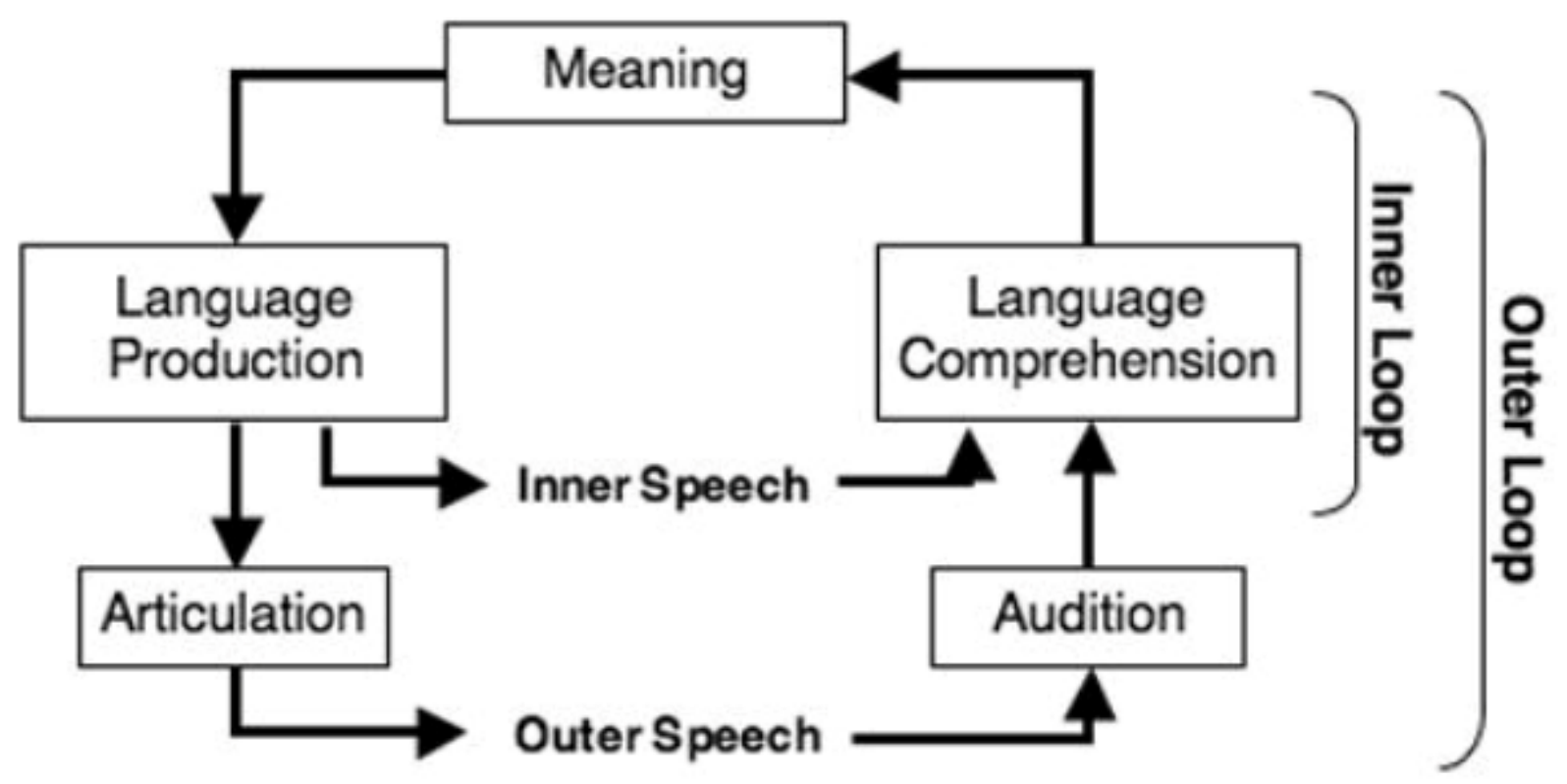

Figura 3: A Teoria do Loop Perceptual e monitoramento de Levelt (Slevec \& Ferreira, 2006). A realimentação do sistema, ou feedback, é reativado pelo discurso interno e externo, a partir de um movimento circular (loop) que integra a produção (output) e a compreensão (input) linguísticas no loop interno, e a articulação (output) e audição (input) no loop externo.

Evidências que sustentam a existência do monitoramento interno são, por exemplo, os casos nos quais alguns erros de fala são corrigidos imediatamente após terem sido produzidos. Isso indica que o erro deve ter sido detectado anteriormente à articulação e os processos de reparação devem ter sido iniciados antes da palavra ser completamente pronunciada. Há também correções feitas no discurso que ocorrem após a pronúncia de apenas o primeiro fonema de uma palavra incorretamente selecionada, por exemplo, “v-horizontal” (Levelt, 1983). Com esse clássico exemplo, é sustentada a hipótese de que um monitoramento interno detecta o erro antes da produção, mas tarde demais para parar a articulação do fonema inicial. Evidências que sustentam a existência do monitoramento externo são mais facilmente observáveis e corrigidas após a produção.

Levelt (1989) descreve que o discurso interior autoproduzido é percebido, analisado e verificado em relação à adequação intencional e contextual e correção linguística. Quando o problema é detectado, a ação corretiva central é tomada. A Teoria do Loop Perceptual de monitoramento do discurso entende que o discurso interior é 
monitorado pelo sistema de compreensão, que detecta erros, comparando a compreensão dos enunciados formulados com aqueles enunciados originalmente pretendidos (Slevec \& Ferreira, 2006). Reivindica-se que o monitor do discurso seja sensível a uma ampla variedade de erros, incluindo erros conceituais, erros de sintaxe, erros lexicais, erros fonêmicos, erros prosódicos, erros de ordem morfológica, erros na adequação do discurso e erros relativos ao contexto social (Levelt, 1989; Postma, 2000). Reivindica-se, ainda, ser ele sensível a quase tudo aquilo a que são sensíveis os ouvintes, o que leva à conclusão de que o monitor utiliza o sistema de compreensão para ouvir tanto o discurso interior quanto o exterior. Em que medida a relação entre o sistema de produção e compreensão do sistema interagem um com o outro, não foi objeto de consideração da abordagem teórica aqui descrita.

Em experimento realizado por Slevec \& Ferreira (2006), verificou-se que indivíduos tinham mais dificuldade em travar a produção de um nome relacionado a uma imagem em resposta a um stop-signal fonologicamente semelhante ao nome da imagem do que em resposta a um stop-signal fonologicamente diferente. No entanto, os indivíduos não demonstraram mais dificuldade em travar a produção em resposta a um sinal de stop que era semanticamente semelhante ao nome de imagem do que a um sinal de stop que era semanticamente diferente. Este padrão de desempenho sustenta a hipótese de que o monitor interior faz comparações com base na informação fonológica, mas é insensível à informação semântica. Um segundo experimento realizado pelos mesmos pesquisadores sustenta a hipótese de que o monitor interno é sensível à semelhança fonológica e não à semelhança semântica, pois mesmo com stop-signals visualmente apresentados, os indivíduos acharam mais difícil parar quando stop-signals eram fonologicamente semelhantes às palavras, mas não quando eram semanticamente semelhantes a elas. Disso conclui-se, com base na Teoria do Loop Perceptual, que o monitor não opera comparação relacionando sistemas de compreensão com sistemas de produção em um nível onde a informação semântica é representada. 


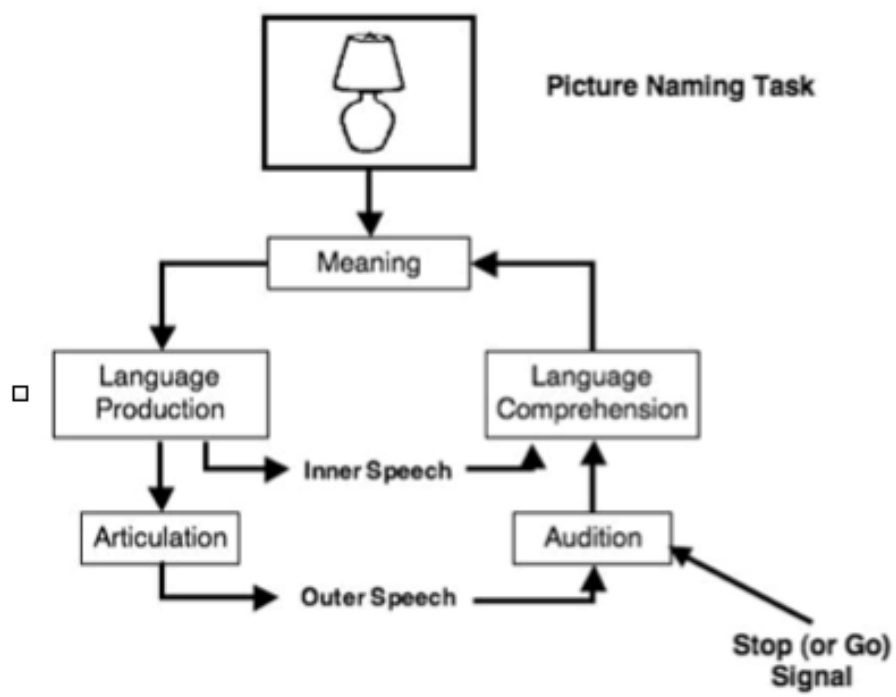

Basic task model, mapped onto Levelt's $(1983,1989)$ perceptual loop theory of speech monitoring. The drawing of a lamp represents the picture-naming task, and is assumed to initiate the word production process. The presentation of the stop-signal or go-signal is intended to 口 "force feed" the comprehension system, mimicking the comprehension of self-produced speech (and thus of the inner monitor).

Figura 4: O modelo de funcionamento de uma tarefa básica segundo a Teoria do Loop Perceptual de Levelt (1983, 1989) (Slevec \& Ferreira, 2006).

A Teoria do Loop Perceptual (Levelt, 1983, 1989) não pressupõe a existência de qualquer dispositivo especial ou mecanismo para detecção de erros. De acordo com esta teoria, os falantes detectam erros em seus discursos, ouvindo a si mesmos. A detecção de erros, em seguida, parte da percepção de que o enunciado produzido é diferente da intenção de fala. Semelhante à detecção de erros na fala de terceiros, os falantes detectam erros em sua própria fala através de um “canal externo” que é o sistema auditivo (Nozari, Dell \& Schwartz, 2011). A teoria padrão de detecção de erros de fala postula que o sistemas de compreensão e produção interagem na busca por erros, mas é o sistema de compreensão que atua sobre o da produção, sendo o monitoramento acionado a partir da compreensão.

Nozari et al. (2011), entretanto, defendem outra linha de pesquisa sobre o mesmo tema. Consideram que a detecção de erros é independente do sistema de compreensão e recaem sobre o sistema de produção. Assumem a possibilidade de existência de vários monitores independentes entre as camadas (layers) do sistema de produção e um loop sensorial ao final, equivalente ao loop externo postulado por Levelt (1983) na Teoria do Loop Perceptual. De acordo com Nozari et al. (2011), o monitoramento baseia-se na produção, segundo qual o processo de produção e monitoramento ocorrem paralelamente e simultaneamente durante todo o processo de produção de enunciado linguístico.

Ainda uma outra corrente teórica é defendida por Pickering \& Garrod (2013). Esses pesquisadores relacionam os sistemas de compreensão e produção como interdependentes no tocante ao monitoramento. Afirmam esses pesquisadores que a produção e a compreensão são sistemas interligados, uma vez que as consideram formas 
de ação e de percepção de ação. Investigaram o processo de produção e compreensão relacionando-os entre si por meio de experimentos de (1) singleword naming, que é tipicamente utilizado na investigação dos processos de compreensão que envolvem produção (Bock, 1996 apud Pickering \& Garrod, 2013) e (2) sentence completion, que é muitas vezes usado para explicar a produção, mas envolve a compreensão (Bock \& Miller, 1991 apud Pickering \& Garrod, 2013). Segundo as pesquisas relatadas os sistemas de produção e compreensão parecem compartilhar alguns circuitos neurais (Scott \& Johns-Rudes, 2003; Wilson et al., 2004 apud Pickering \& Garrod, 2013). Por exemplo, Paus et al. (1996 apud Pickering \& Garrod, 2013) encontraram ativação de regiões associadas com a percepção da fala quando pessoas sussurravam, mas não podiam ouvir suas próprias vozes. Ouvintes também ativaram os músculos apropriados na língua e lábios enquanto escutavam algum tipo de fala, mas o mesmo não aconteceu em experimento de nonspeech (Fadiga et al., 2002; Watkins et al., 2003 apud Pickering \& Garrod, 2013). Além disso, o aumento da atividade muscular nos lábios está associada ao aumento da atividade do fluxo sanguíneo na área de Broca, sugerindo que esta área faz mediação entre os sistemas de compreensão e produção na percepção da fala (Watkins \& Paus, 2004 apud Pickering \& Garrod, 2013).

\section{4) Críticas}

Críticas feitas à Teoria do Loop Perceptual focalizam seu foco na compreensão (Nozari, Dell \& Schwartz, 2011). Estudos realizados com afásicos mostrou que alguns pacientes têm um sistema de compreensão intacto, mas o monitoramento prejudicado ou vice-versa. Isto indica que o sistema de compreensão pode não ser crucial para o monitoramento quando assumida a possibilidade de serem os dois módulos linguísticos encapsulados, que não dependem um do outro. Evidência acerca da dissociação entre a compreensão e detecção de erro veio também a partir de estudos de pacientes afásicos, que mostraram detecção de erros pobres, apesar da boa compreensão. Há relatos de resultados semelhantes em doentes de Parkinson, que não detectaram $75 \%$ de erros (uma taxa comparável a de pacientes de Alzheimer com má compreensão), apesar de terem demonstrado boa capacidade de compreensão. No entanto, ainda pacientes dotados de boa compreensão, mas baixa eficácia de automonitoramento, parecem ser capazes de detectar erros na fala de terceiros perfeitamente.

Outra crítica é relacionada ao fato de que não há dúvida de que os seres humanos são capazes de monitorar seu discurso interior quando eles não estão falando em voz alta (Nozari, Dell \& Schwartz, 2011). Isto significaria que o monitoramento poderia presumivelmente ocorrer durante a produção da fala. Huettig \& Hartsuiker (2010 apud Nozari, Dell \& Schwartz, 2011) testaram a hipótese de que o discurso interior é monitorado quando há produção de fala por meio de experimento de eyetracking. Eles registraram os movimentos dos olhos dos participantes enquanto nomeavam uma imagem (por exemplo, heart) na presença de um concorrente fonológico (por exemplo, harp) e duas outras palavras não relacionadas. Dada a 
semelhança fonológica do concorrente com o alvo, era esperado que houvesse fixação temporária sobre o concorrente. A lógica embutida no experimento seria de que se o discurso interior é percebido durante a produção, seria de esperar que a imagem concorrente (harp) atraísse mais olhares do que imagens não relacionadas num curto espaço de tempo (+50 ms). Mas se apenas a fala plena, no estágio articulatório, é monitorada, o ponto de registro de tempo mais curto para os participantes fixarem no concorrente fonológico seria em torno de 300 ms pós-início da fala. Os dados foram compatíveis com a segunda possibilidade. Os participantes começaram a fixar o concorrente fonológico 350-500 ms depois que eles passaram a nomear o alvo. Ou seja, não havia nenhuma indicação de que monitoraram seu discurso interior enquanto o enunciado era produzido, mas apenas depois de sua articulação plena.

Ainda encontramos críticas à teoria do loop perceptual indicando que essa abordagem não é restrita o suficiente para gerar facilmente predições testáveis. Não é claro exatamente que elementos do discurso podem ser monitorados, quando esse monitoramento ocorre, nem em que nível de representação mental o monitoramento ocorre.

\section{5) Monitoramento da escrita}

$\mathrm{Na}$ área de produção escrita, a literatura específica atribui ao monitoramento os mesmos valores de deteç̧ão de erros e correção aplicados ao monitorando da fala ao processo de revisão e feedback.

Como na produção da fala, a produção da escrita é complexa, envolvendo uma série de processos cognitivos, linguísticos e motores. Em ambos os casos, os processos cognitivos subjacentes compartilham características na medida em que informação de natureza lexical, sintática e semântica disponível para a produção escrita passam pelas mesmas etapas de conceptualização da mensagem tal qual proposto por Levelt na Teoria do Loop Perceptual $(1983,1989)$ até ser encaminhado para o sistema de desempenho verbal.

Mas diferentemente do que ocorre na produção da fala, na escrita, o processo de revisão do sistema de produção é realimentado apenas pelo próprio escritor em diálogo consigo mesmo e com o próprio texto produzido até o momento. O processo de revisão serve ao controle corretivo de monitoramento diretamente. Ao detectar um erro em um contexto comunicativo, novas informações armazenadas na memória são acessadas para realimentação do sistema percentual da mesma forma descrita na Teoria do Loop Perceptual de Levelt $(1983,1989)$. Podemos inferir que o feedback desempenha papel relevante no acesso à memória enquanto “fio condutor" que encaminha ao sistema executivo central o tipo de informação incongruente (entre representação mental e texto até então produzido) e percebida na fase de detecção de erros e para satisfação da qual será necessário acessar bases de dados de memória para realimentação do sistema de revisão. Assume-se, portanto, que o monitoramento e o feedback são diretamente interligados. 
O monitoramento relaciona-se, conforme já visto, aos sistemas de percepção e atenção, pressupostos básicos psicolinguísticos, e estão inseridos nos sistemas de processamento de informação que fundamentam a base da pesquisa em cognição e escrita. Por meio de metodologia tecnológica com uso de rastreamento ocular e movimentação do cursor e teclado, a pesquisa relacionada ao monitoramento na produção escrita focaliza o sistema de revisão com análise dos dados de pausas, segmentações, localização destas e tipos de inserções e correções feitas no texto discursivo.

Por meio do registro dos dados cognitivos capturados, pode-se monitorar os focos de atenção do participante em experimentos de produção. Para Rayner (1998), a metodologia de eyetracking permite capturar dados cognitivos quantificáveis do processo de monitoramento e, combinada com a metodologia de registro de movimentação do teclado, pode prover dados mais granulares ainda sobre a localidade, a distribuição, as segmentações indicadoras de interrupções e que tipo de elemento é introduzido ao discurso em seguida à interrupção/segmentação e duração de fixações como indicativos do tipo de atividade cognitiva em processamento (Griffin, 2001).

\section{6) Conclusão}

Pesquisas que focalizam o monitoramento à luz do processamento cognitivo tem tomado mais força há alguns anos e vem contribuindo significativamente para elucidar questões sobre o papel por ele desempenhado. Essas pesquisas tem focalizado em na metodologia tecnológica sofisticada e nos remetem, de várias formas, às mesmas questões pontuadas por Levelt $(1983,1989)$.

Certamente o estudo com base em medidas cognitivas coletadas via rastreamento ocular e movimentação do teclado virão a ser ainda mais informativas quanto mais pesquisas dediquem-se a refiná-las.

7) Referências Bibliográficas

BADDELEY, A.D. \& HITCH, G.J. "Working Memory”. In: BOWER, G.A. (ed.), Recent Advances in Learning and Motivation, vol. 8. New York: Academic Press, 1974, pp. 47-89. 
BADDELEY, A.D., GATHERCOLE, S.E. \& PAPAGNO, C. (1998). "The Phonological Loop as a Language Learning Device”. Psychological Review 105(1), pp. 158-173.

ERICSSON, K.A. \& KINTSCH, W. (1991). "Memory in Comprehension and Problem Solving: a Long Term Working Memory. Institute of Cognitive Science, pp. 91-113.

FERREIRA V.S. (2010). "Language production". Wiley Interdisciplinary Reviews: Cognitive Science. 1, 834-844.

GRIFFIN, Z.M. (2001). "Gaze Durations uring speech reflect word selection and phonological encoding”. Cognition, 82, B1-B14.

LEVELT, W. J. M. (1983). “Monitoring and self-repair in speech”. Cognition 14, pp. 41-104.

LEVELT, W. J. M. Speaking From Intention to Articulation. Cambridge: MIT Press, 1989.

NOZARI, N., DELL, G. S., \& SCHWARTZ, M. F. (2011). Is comprehension necessary for error detection? A conflict-based account of monitoring in speech production. Cognitive Psychology. 63, 1-33.

PICKERING, M.J. \& GARROD, S. (2013). “An integrated theory of language production and comprehension”. Behavioral And Brain Sciences 36, pp. 329-392.

POSTMA, A. (2000). "Detection of errors during speech production: a review of speech monitoring models”. Cognition 77(2), pp. 97-131.

SLEVEC, L.R. \& FERREIRA, V.S. (2006). "Halting in Single Word Production: A Test of the Perceptual Loop Theory of Speech Monitoring”. Journal Memory Language 54(4), pp. 515-540. 